Communications in Physics, Vol. 29, No. 3 (2019), pp. 223-240

DOI:10.15625/0868-3166/29/3/13742

\title{
GENERATION OF MAXIMALLY ENTANGLED STATES BY A KERR-LIKE NONLINEAR COUPLER INTERACTING WITH EXTERNAL FIELDS
}

\author{
DOAN QUOC KHOA ${ }^{1, \dagger}$, LUONG THI TU OANH ${ }^{2,3}$, CHU VAN LANH $^{2}$, \\ NGUYEN THI DUNG ${ }^{4}$ AND DO HONG SON ${ }^{4}$
}

${ }^{1}$ Quang Tri Teacher Training College, Dong Ha, Quang Tri, Vietnam

${ }^{2}$ Vinh University, 182 Le Duan, Vinh, Vietnam

${ }^{3} \mathrm{Nghe}$ An College of Education, Vinh, Vietnam

${ }^{4}$ Hong Duc University, Thanh Hoa, Viet Nam

${ }^{\dagger}$ E-mail: khoa_dqspqt@yahoo.com

Received 9 April 2019

Accepted for publication 22 July 2019

Published 15 August 2019

\begin{abstract}
We study a model with two nonlinear oscillators (Kerr-like nonlinear coupler) pumped by two external coherent fields. Using the formalism of nonlinear quantum scissors (NQS) introduced before for quantum state engineering, we obtain the wave function describing the evolution of the system as a combination of n-photon Fock states. Considered NQS generates a truncation of optical states that leads to achieve two-qubit states due to the nonlinear properties of oscillators and their interaction. Solving a set of coupled stochastic integro-differential equations involved in the problem with all initial conditions, we achieve analytical formulae for the complex probability amplitudes of n-photon states. In particular, evolution of the system generates maximally entangled states as so-called Bell-like states. We consider our model for both damping and without damping cases and compare these results with that achieved previously in the literature.
\end{abstract}

Keywords: Kerr-like nonlinear coupler, Bell-like states, entropy of entanglement.

Classification numbers: 03.65.Ud, 42.50.Dv.

(C)2019 Vietnam Academy of Science and Technology 


\section{INTRODUCTION}

Entanglement is an important information resource in quantum computing [1,2]. It is a fundamental element for most of algorithms in quantum information theory such as quantum teleportation, quantum cryptography, superdense coding, quantum error correction. Indeed, the resources needed to implement a particular algorithm of quantum information are strictly related to the entanglement properties of the states involved in the considered algorithm. Therefore, it is highly desirable to consider different possibilities of entanglement creation. Depending on the choice of physical systems to be implemented the entangled states can be generated in various ways, for example using spin systems, trapped ions [3], quantum dots [4], Bose-Einstein condensates [5]. Generally, any multi-state system can be implemented to create entanglement. Entangled states can be also found in optical fields through mutual interactions among their modes. Then quantum entanglement can be generated in photon-number states [6]. The last one is the most promising due to the fast progress in building photon-number resolving detectors based on different mechanisms [7-11].

One of the most effective tools to do this is so-called nonlinear quantum scissors (NQS). They are defined as optical devices in which nonlinear elements (for example nonlinear oscillators) are used (see the review paper [12] and the references quoted therein). As it has been emphasized there, the quantum entanglement is one of the most essential problems of the current quantum theory and it is widely accepted recently that its solution will contribute to a deep understanding of quantum world. Therefore, the creation of the finite-dimensional and truncated states, which are strictly related to the entanglement problems plays an important role in answering the fundamental questions of the quantum theory.

In recent papers, one develops the idea of NQS device for two-mode state, which has been initiated in $[13,14]$. The considered model consists of two quantum oscillators described by Kerrlike nonlinearities. Various kinds of coupling between the oscillators have already been studied among them as linear [13], nonlinear [14] or parametric [15]. The systems were enlarged for three Kerr-like nonlinear oscillators coupling with each other and being pumped by external coherent fields in two modes [16]. A family of states depicting three-qubit systems in a context of quantum steering phenomena [17] and the system of three interacting qubits is investigated [18]. The timedependence of the interaction energy resulting in the Kerr-like behaviour of the second-harmonic generation in the far-off resonant regime from the oscillations is researched [19]. It has been shown that the considered model can generate finite dimensional quantum states, so as a result, one can obtain one-, two- or three-dimensional truncated Fock states. While system dynamics is restricted to states of a few modes, there is only a possibility for generation of maximally entangled states (Bell-like states). These states have been introduced by John S. Bell and related to his famous inequality [20] for discussion of the well-known Einstein-Podolsky-Rosen paradox [21], which leads to the understanding of the present quantum theory. Due to this fact they are sometimes called EPR pairs, which are fundamental tools in quantum computing. For the description of the Bell states and the discussion concerning their properties and applications see, for example, [22$27]$ and the references quoted therein.

In the previous works of $[14,28]$, one considered a Kerr-like nonlinear coupler in which two nonlinear oscillators are nonlinearly coupled with each other and one [14] or two [28] of these nonlinear oscillators interact with the external coherent fields. We now extend our consideration 
to the case of two nonlinear oscillators interacting with two external coherent fields and study the evolution equation involved in the problem with all initial conditions for arbitrary strength of coupling between the modes with external coherent fields. It has been already recognized in [13] that the system with the initial Fock states is much more experimentally challenging than assuming initially the vacuum states only. This aspect has been analyzed in detail in [12]. It follows from discussions there that despite the fact of nonperfect initial state preparation, there is still a nonvanishing probability that the truncated state will have the desired form.

\section{THE KERR-LIKE NONLINEAR COUPLER PUMPED IN TWO MODES}

The model of the Kerr-like nonlinear coupler which is examined here includes two nonlinear oscillators that are characterized by Kerr nonlinearities $\chi_{a}$ and $\chi_{b}$ with the field modes $a$ and $b$, respectively. These nonlinear oscillators are nonlinearly coupled to each other and both are pumped by two external coherent fields. In the interaction picture, this system can be described by the Hamiltonian as

$$
\hat{H}=\hat{H}_{1}+\hat{H}_{2}+\hat{H}_{3}+\hat{H}_{4}
$$

in which

$$
\begin{aligned}
& \hat{H}_{1}=\frac{\chi_{a}}{2}\left(\hat{a}^{\dagger}\right)^{2} \hat{a}^{2}, \\
& \hat{H}_{2}=\frac{\chi_{b}}{2}\left(\hat{b}^{\dagger}\right)^{2} \hat{b}^{2}, \\
& \hat{H}_{3}=\epsilon\left(\hat{a}^{\dagger}\right)^{2} \hat{b}^{2}+\epsilon^{*}\left(\hat{b}^{\dagger}\right)^{2} \hat{a}^{2}, \\
& \hat{H}_{4}=\alpha \hat{a}^{\dagger}+\alpha^{*} \hat{a}+\beta \hat{b}^{\dagger}+\beta^{*} \hat{b},
\end{aligned}
$$

where $\hat{H}_{1}, \hat{H}_{2}$ depict nonlinear oscillators in two modes $a$ and $b$, respectively. $\hat{H}_{3}$ is a term of Hamiltonian which depicts interaction between the modes and $\hat{H}_{4}$ corresponds to interaction of the modes with external coherent fields. $\hat{a}$ and $\hat{b}$ are boson annihilation operators and $\hat{a}^{\dagger}$ and $\hat{b}^{\dagger}$ are boson creation operators corresponding to two modes $a$ and $b$, respectively. Complex parameters $\alpha$ and $\beta$ depict strengths of coupling between the modes $a$ and $b$ with external coherent fields, respectively. The parameter $\epsilon$ is a constant that describes the strength of internal interaction between two oscillators in the system. It should be noted that $\hat{H}_{3}$ does not have the terms containing $\hat{a}^{\dagger} \hat{a} \hat{b}^{\dagger} \hat{b}$ element and their combination.

We here limit our model to the case without damping. Then the time-dependent wave function depicting the evolution of the system is defined by the Schrödinger equation in interaction picture:

$$
i \frac{d}{d t}|\psi(t)\rangle=\hat{H}|\psi(t)\rangle
$$

where wave function $|\psi(t)\rangle$ depicting the evolution of the system is expanded in the n-photon Fock states, it has the following form

$$
|\psi(t)\rangle=\sum_{k, l=0}^{\infty} c_{k l}(t)|k\rangle_{a}|l\rangle_{b},
$$


where $c_{k l}(t)$ are complex probability amplitudes. From the expressions (6) and (7) we obtain motion equations for $c_{k l}(t)$ (use units $\hbar=1$ ) as

$$
\begin{aligned}
i \frac{d}{d t} c_{k l}(t)= & {\left[\frac{1}{2} \chi_{a} k(k-1)+\frac{1}{2} \chi_{b} l(l-1)\right] c_{k l}(t) } \\
& +\varepsilon \sqrt{(l+2)(l+1) k(k-1)} c_{k-2, l+2}(t) \\
& +\varepsilon^{*} \sqrt{(k+2)(k+1) l(l-1)} c_{k+2, l-2}(t) \\
& +\alpha^{*} \sqrt{k+1} c_{k+1, l}(t)+\alpha \sqrt{k} c_{k-1, l}(t) \\
& +\beta^{*} \sqrt{l+1} c_{k, l+1}(t)+\beta \sqrt{l} c_{k, l-1}(t) .
\end{aligned}
$$

We will use the formalism of NQS as in [14]. Then the wave function (7) can be truncated to the wave function which is depicted by only some Fock states of an infinite number of photons. Thence the wave function of system can be expressed only in a set of four states: $|0\rangle_{a}|2\rangle_{b},|1\rangle_{a}|2\rangle_{b}$, $|2\rangle_{a}|1\rangle_{b}$ and $|2\rangle_{a}|0\rangle_{b}$ with the following form

$$
\begin{aligned}
|\psi(t)\rangle_{c u t} & =c_{02}^{(m n)}(t)|0\rangle_{a}|2\rangle_{b}+c_{12}^{(m n)}(t)|1\rangle_{a}|2\rangle_{b} \\
& +c_{21}^{(m n)}(t)|2\rangle_{a}|1\rangle_{b}+c_{20}^{(m n)}(t)|2\rangle_{a}|0\rangle_{b},
\end{aligned}
$$

in wich $m, n=0,1,2$ are the notation of oscillator modes that are initially in states $|m\rangle_{a}|n\rangle_{b}$. For general case, substituting these values into (8), the equations for complex probability amplitudes $c_{02}^{(m n)}(t), c_{12}^{(m n)}(t), c_{21}^{(m n)}(t)$ and $c_{20}^{(m n)}(t)$ have the following form

$$
\begin{aligned}
i \frac{d}{d t} c_{02}^{(m n)}(t) & =2 \varepsilon^{*} c_{20}^{(m n)}(t)+\alpha^{*} c_{12}^{(m n)}(t), \\
i \frac{d}{d t} c_{12}^{(m n)}(t) & =\alpha c_{02}^{(m n)}(t), \\
i \frac{d}{d t} c_{21}^{(m n)}(t) & =\beta c_{20}^{(m n)}(t), \\
i \frac{d}{d t} c_{20}^{(m n)}(t) & =2 \varepsilon c_{02}^{(m n)}(t)+\beta c_{21}^{(m n)}(t) .
\end{aligned}
$$

In this work, equations (10) will be solved for all four initial states $|2\rangle_{a}|0\rangle_{b},|0\rangle_{a}|2\rangle_{b}$, $|2\rangle_{a}|1\rangle_{b}$ and $|1\rangle_{a}|2\rangle_{b}$ in which we assume that $\alpha, \beta$ and $\varepsilon$ have the real values. For simplicity and convenience in comparing our results with those found earlier, we only present here analytical solutions of complex probability amplitudes for the initial state $|2\rangle_{a}|0\rangle_{b}$ as follows

$$
\begin{aligned}
c_{20}^{(20)}(t)= & \frac{1}{16 i \lambda^{2}}\left[\frac{\lambda+\alpha^{2}-\beta^{2}-4 \varepsilon^{2}}{\mu_{1}}\left(\left(\mu-4 \mu_{1} \lambda-4 \mu_{2} \lambda\right) \sin \left(\mu_{1} t\right)+8 i \mu_{1} \lambda \cos \left(\mu_{1} t\right)\right)\right. \\
& \left.+\frac{\lambda-\alpha^{2}+\beta^{2}+4 \varepsilon^{2}}{\mu_{2}}\left(\left(\mu-4 \mu_{1} \lambda-4 \mu_{2} \lambda\right) \sin \left(\mu_{2} t\right)+8 i \mu_{2} \lambda \cos \left(\mu_{2} t\right)\right)\right],
\end{aligned}
$$




$$
\begin{aligned}
c_{21}^{(20)}(t)= & -\frac{1}{16 \beta \lambda^{2}}\left[\left(\lambda-\alpha^{2}+\beta^{2}+4 \varepsilon^{2}\right)\left(\left(\mu-4 \mu_{1} \lambda-4 \mu_{2} \lambda\right) \cos \left(\mu_{2} t\right)+8 i \mu_{1} \lambda \sin \left(\mu_{2} t\right)\right)\right. \\
& \left.+\left(\lambda+\alpha^{2}-\beta^{2}-4 \varepsilon^{2}\right)\left(\left(\mu-4 \mu_{1} \lambda-4 \mu_{2} \lambda\right) \cos \left(\mu_{1} t\right)+8 i \mu_{2} \lambda \sin \left(\mu_{1} t\right)\right)\right], \\
c_{12}^{(20)}(t)= & \frac{\alpha \varepsilon}{4 i \lambda^{2}}\left[\frac{\mu-4 \mu_{1} \lambda-4 \mu_{2} \lambda}{\mu_{1}} \sin \left(\mu_{1} t\right)+\frac{\mu-4 \mu_{1} \lambda-4 \mu_{2} \lambda}{\mu_{2}} \sin \left(\mu_{2} t\right)\right. \\
& \left.-8 i \lambda\left(\cos \left(\mu_{1} t\right)-\cos \left(\mu_{2} t\right)\right)\right], \\
c_{02}^{(20)}(t)= & \frac{\varepsilon}{4 \lambda^{2}}\left[\left(\mu-4 \mu_{1} \lambda-4 \mu_{2} \lambda\right) \cos \left(\mu_{1} t\right)+\left(\mu-4 \mu_{1} \lambda-4 \mu_{2} \lambda\right) \cos \left(\mu_{2} t\right)\right. \\
& \left.+8 i \lambda\left(\mu_{1} \sin \left(\mu_{1} t\right)-\mu_{2} \sin \left(\mu_{2} t\right)\right)\right],
\end{aligned}
$$

where

$$
\begin{aligned}
& \lambda=\sqrt{\left(\alpha^{2}+2 \alpha \beta+\beta^{2}+4 \epsilon^{2}\right)\left(\alpha^{2}+2 \alpha \beta+\beta^{2}+4 \epsilon^{2}\right)}, \\
& \mu=-8\left(\mu_{1}^{3}-\mu_{2}^{3}\right)+4 \alpha^{2}\left(\mu_{1}-\mu_{2}\right)+4 \beta^{2}\left(\mu_{1}-\mu_{2}\right)+16 \varepsilon^{2}\left(\mu_{1}-\mu_{2}\right), \\
& \mu_{1}=\frac{1}{2} \sqrt{2 \alpha^{2}+2 \beta^{2}+8 \epsilon^{2}-2 \lambda}, \\
& \mu_{2}=\frac{1}{2} \sqrt{2 \alpha^{2}+2 \beta^{2}+8 \epsilon^{2}+2 \lambda} .
\end{aligned}
$$

For the case of one nonlinear oscillator pumped by an external coherent field $(\beta=0)$, our results become exactly the same as those in [14]. Moreover, for the case $\alpha=\beta$, our result becomes exactly the same as that obtained in [28].

To show the worth of our analytical method, we calculate the fidelity of output state by numerical calculation in which the initial input state is $|2\rangle_{a}|j\rangle_{b}, \mathrm{j}=0,1$. Then the time-evolution of output wave function $|\psi(t)\rangle$ will have the following form

$$
|\psi(t)\rangle=\exp (-i \hat{H} t)|2\rangle_{a}|j\rangle_{b}
$$

The fidelity of output state will be calculated by the definition

$$
F\left(\hat{\rho}, \hat{\rho}_{\text {cut }}\right)=\left\{\operatorname{Tr}\left[\left(\sqrt{\hat{\rho}_{\text {cut }}} \hat{\rho} \sqrt{\hat{\rho}_{\text {cut }}}\right)^{\frac{1}{2}}\right]\right\}^{2}
$$

where

$$
\begin{aligned}
& \hat{\rho}=|\psi(t)\rangle\langle\psi(t)|, \\
& \hat{\rho}_{\text {cut }}=|\psi(t)\rangle_{\text {cutcut }}\langle\psi(t)| .
\end{aligned}
$$

The numerical results of time-evolution of fidelity are shown in Fig. 1. It is worth to use this analytical method because we can see that the fidelity of output state is unit approximation. The variabilities of time-evolution of the deviation of fidelity from unit (1-F) are always smaller than $1.2 \times 10^{-3}$ at all moments of times. Similarly for the the initial state $|2\rangle_{a}|1\rangle_{b}$, the fidelity is a little smaller than fidelity of the case that is generated by initial state $|2\rangle_{a}|0\rangle_{b}$ in [28]. 


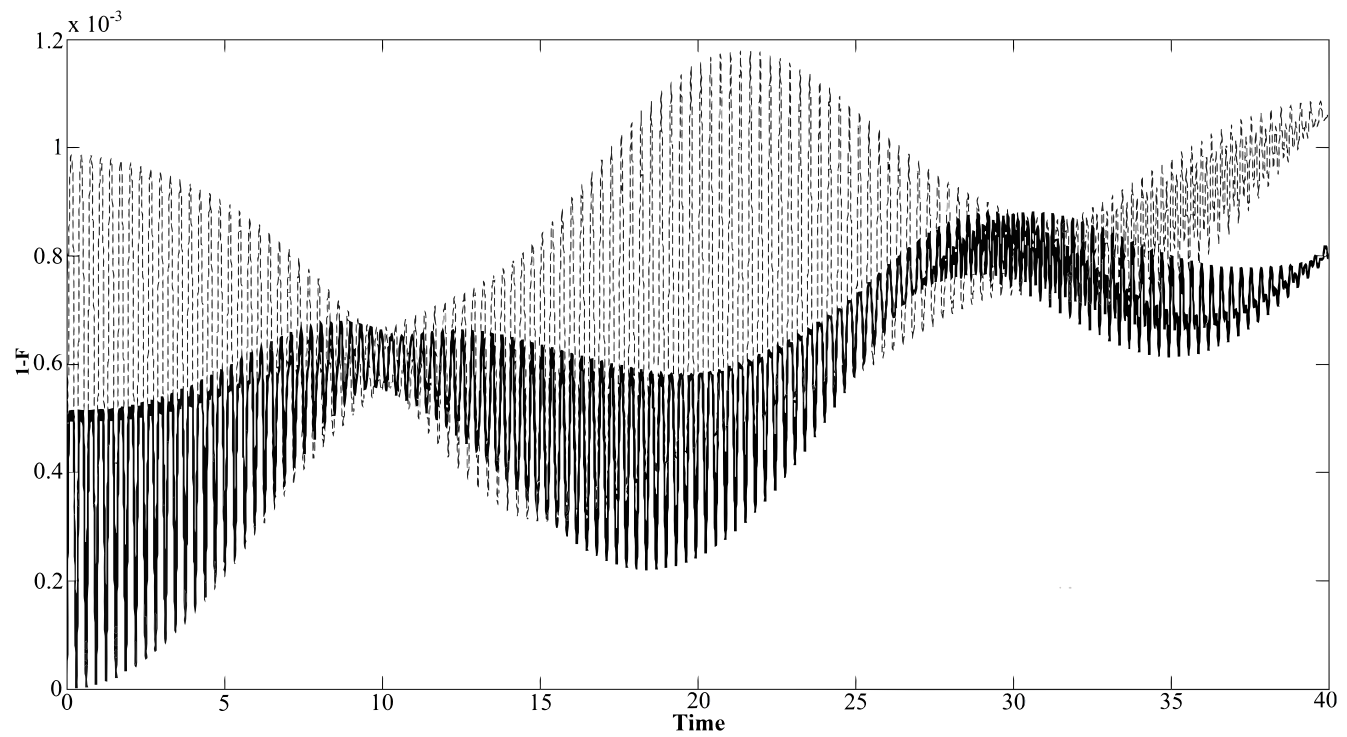

Fig. 1. The time-evolution of 1-F corresponding to the initial conditions $|2\rangle_{a}|0\rangle_{b}$ (solid curve) and $|2\rangle_{a}|1\rangle_{b}$ (dashed line). The nonlinearities $\chi_{a}=\chi_{b}=20 \mathrm{rad} / \mathrm{s}$, the coupling strengths $\alpha=\beta=\frac{\pi}{20} \mathrm{rad} / \mathrm{s}, \varepsilon=\frac{\pi}{80} \mathrm{rad} / \mathrm{s}$. Time unit is scalled in $1 / \chi$.

\section{GENERATION OF MAXIMALLY ENTANGLED STATES}

This section describes maximally entangled states, the output states $|\psi(t)\rangle_{\text {cut }}$ that are generated by our model. To find these states, we depict time-evolution of entanglement of the system in terms of the von Neumann entropy, which has been defined in $[28,29]$. From the expression (9) and the full density matrix $\rho_{a b}=|\psi\rangle_{\text {cutcut }}\langle\psi|$, we receive the partial trace of $\rho_{a b}$ with respect to the mode $b$ in the form

$$
\begin{aligned}
\rho_{b}=\operatorname{Tr}_{a} \rho_{a b} & =\left|c_{20}^{(m n)}\right|^{2}|0\rangle_{b b}\left\langle 0\left|+c_{20}^{(m n)} c_{21}^{(m n) *}\right| 0\right\rangle_{b b}\left\langle 1\left|+c_{21}^{(m n)} c_{20}^{(m n) *}\right| 1\right\rangle_{b b}\langle 0| \\
& +\left|c_{21}^{(m n)}\right|^{2}|1\rangle_{b b}\left\langle 1\left|+\left(\left|c_{02}^{(m n)}\right|^{2}+\left|c_{12}^{(m n)}\right|^{2}\right)\right| 2\right\rangle_{b b}\langle 2| .
\end{aligned}
$$

Then, one of the entanglement measures of the system is the von Neumann entropy which has the following form

$$
E=-\operatorname{Tr} \rho_{a} \log _{2} \rho_{a}=-\operatorname{Tr} \rho_{b} \log _{2} \rho_{b}=-\lambda_{1} \log _{2} \lambda_{1}-\lambda_{2} \log _{2} \lambda_{2},
$$

in which $\lambda_{1}$ and $\lambda_{2}$ are eigenvalues of $\rho_{b}$. The entropy of entanglement changes its value from zero for separable states to 1 ebit for the maximally entangled states.

We shall now express the derived wave function in the basic Bell states with the following form

$$
|\psi\rangle=\sum_{i=1}^{8} b_{i}\left|B_{i}\right\rangle
$$



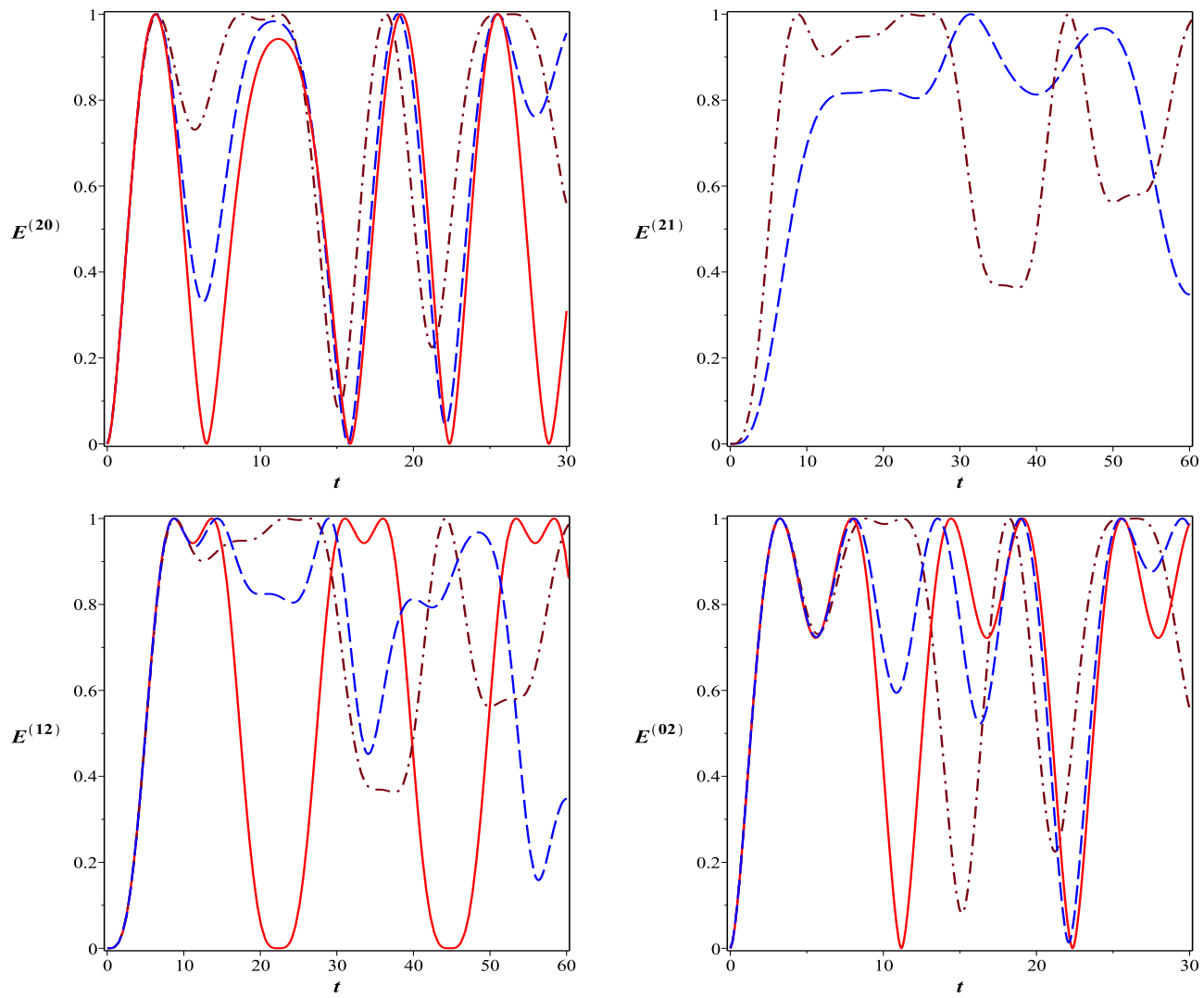

Fig. 2. Evolution of the entropies of entanglement of the truncated states corresponding to the initial states $|2\rangle_{a}|0\rangle_{b}\left(E^{(20)}\right),|2\rangle_{a}|1\rangle_{b}\left(E^{(21)}\right),|1\rangle_{a}|2\rangle_{b}\left(E^{(12)}\right)$ and $|0\rangle_{a}|2\rangle_{b}\left(E^{(02)}\right)$ with $\alpha=\pi / 25, \varepsilon=\pi / 25$. Solid curve is for $\beta=0$, dashed curve is for $\beta=\pi / 50$ and dashed dotted curve is for $\beta=\pi / 25$. The time is scaled in $1 / \chi$ units.

where $\left|B_{i}\right\rangle$ are maximally entangled states that can be expressed as functions of the Fock states examined here:

$$
\begin{aligned}
\left|B_{1}\right\rangle=\frac{1}{\sqrt{2}}\left(|2\rangle_{a}|0\rangle_{b}+i|0\rangle_{a}|2\rangle_{b}\right), & \left|B_{2}\right\rangle=\frac{1}{\sqrt{2}}\left(|2\rangle_{a}|0\rangle_{b}-i|0\rangle_{a}|2\rangle_{b}\right), \\
\left|B_{3}\right\rangle=\frac{1}{\sqrt{2}}\left(|2\rangle_{a}|0\rangle_{b}+i|1\rangle_{a}|2\rangle_{b}\right), & \left|B_{4}\right\rangle=\frac{1}{\sqrt{2}}\left(|2\rangle_{a}|0\rangle_{b}-i|1\rangle_{a}|2\rangle_{b}\right), \\
\left|B_{5}\right\rangle=\frac{1}{\sqrt{2}}\left(|2\rangle_{a}|0\rangle_{b}+|1\rangle_{a}|2\rangle_{b}\right), & \left|B_{6}\right\rangle=\frac{1}{\sqrt{2}}\left(|2\rangle_{a}|0\rangle_{b}-|1\rangle_{a}|2\rangle_{b}\right), \\
\left|B_{7}\right\rangle=\frac{1}{\sqrt{2}}\left(|0\rangle_{a}|2\rangle_{b}+|2\rangle_{a}|1\rangle_{b}\right), & \left|B_{8}\right\rangle=\frac{1}{\sqrt{2}}\left(|0\rangle_{a}|2\rangle_{b}-|2\rangle_{a}|1\rangle_{b}\right) .
\end{aligned}
$$

Figure 2 shows the depending of time-evolution of entangled entropies $E^{(20)}, E^{(21)}, E^{(12)}$ and $E^{(02)}$ for different values of coupling strength $\beta$ with four initial states are $|2\rangle_{a}|0\rangle_{b},|2\rangle_{a}|1\rangle_{b}$, $|1\rangle_{a}|2\rangle_{b},|0\rangle_{a}|2\rangle_{b}$, respectively. The entanglement of output state in this figure confirms that the 
maximum values of entanglement depend on the values of strength of coupling between two modes with external coherent fields. When $\beta=0$, the peaks of entangled entropies appear as a periodic variability. Entropy of entanglement $E^{(20)}$ becomes exactly the same as the results obtained in [14]. As for the values of the peaks of $E^{(12)}$ and $E^{(02)}$, all of them are approximately equal to 1 . When $\beta \neq 0$, the second peak of $E^{(20)}$ increases, whereas the values of some peaks of $E^{(12)}$ and $E^{(21)}$ decrease and the quantum state are always in entangled states. When $\beta$ increases, the second peak of $E^{(20)}$ splits into two peaks and they reach approximately values equal to units and the periodic of entangled entropies changes. Thus, the presence of $\beta$ changes the values and positions of the peaks of entangled entropies. Moreover, in the same interval of time, the output state leads maximum values of entropies of entanglement $E^{(02)}$ and $E^{(12)}$ at more moments of time than the results that were mentioned in [14].
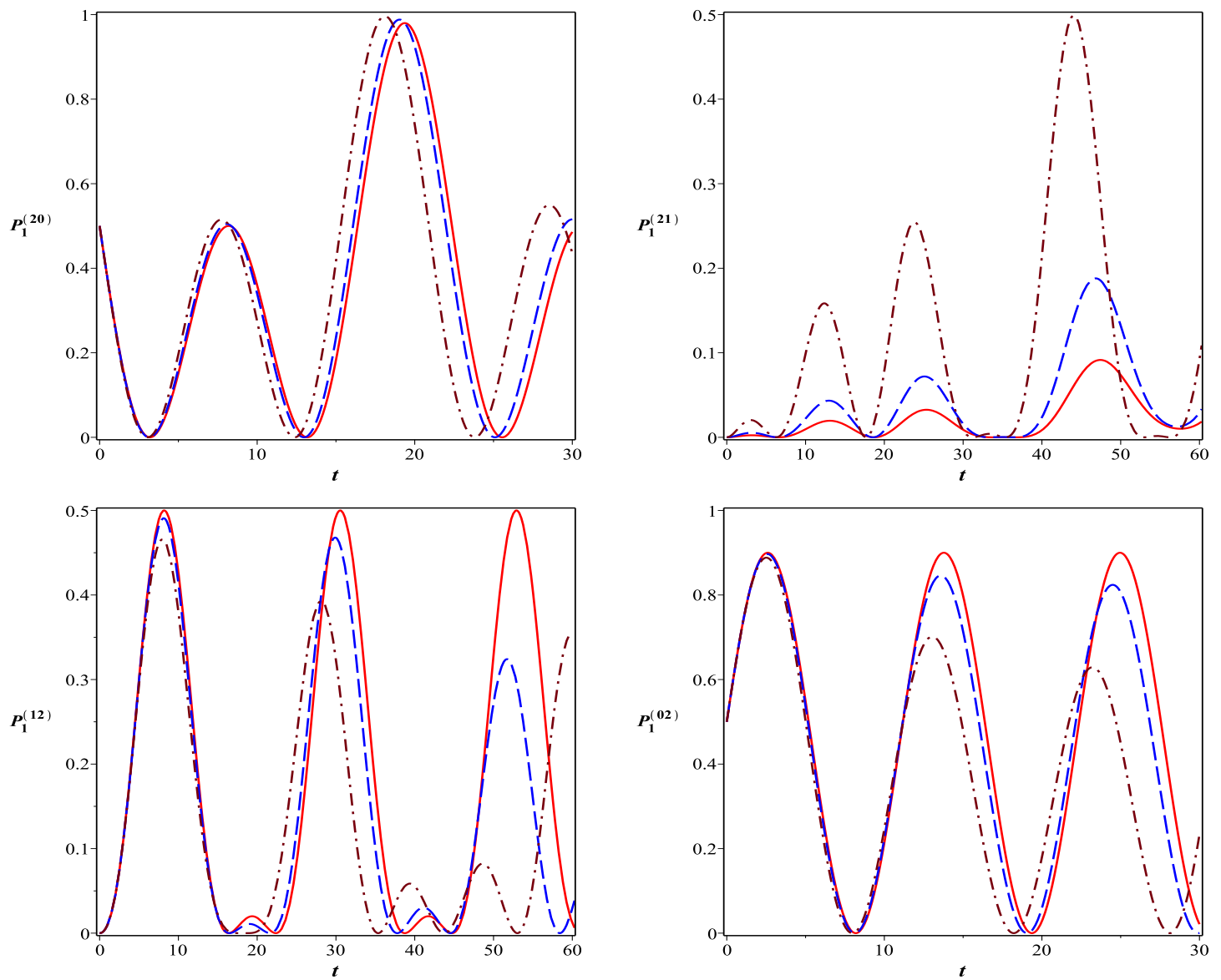

Fig. 3. The probabilities that the system exists in the Bell-like $\left|B_{1}\right\rangle$ corresponding to the initial states $|2\rangle_{a}|0\rangle_{b}\left(P_{1}^{(20)}\right),|2\rangle_{a}|1\rangle_{b}\left(P_{1}^{(21)}\right),|1\rangle_{a}|2\rangle_{b}\left(P_{1}^{(12)}\right)$ and $|0\rangle_{a}|2\rangle_{b}\left(P_{1}^{(02)}\right)$ with $\alpha=\pi / 25, \varepsilon=\pi / 25$. Solid curve is for $\beta=0$, dashed curve is for $\beta=\pi / 50$ and dashed dotted curve is for $\beta=\pi / 25$. The time is scaled in $1 / \chi$ units. 

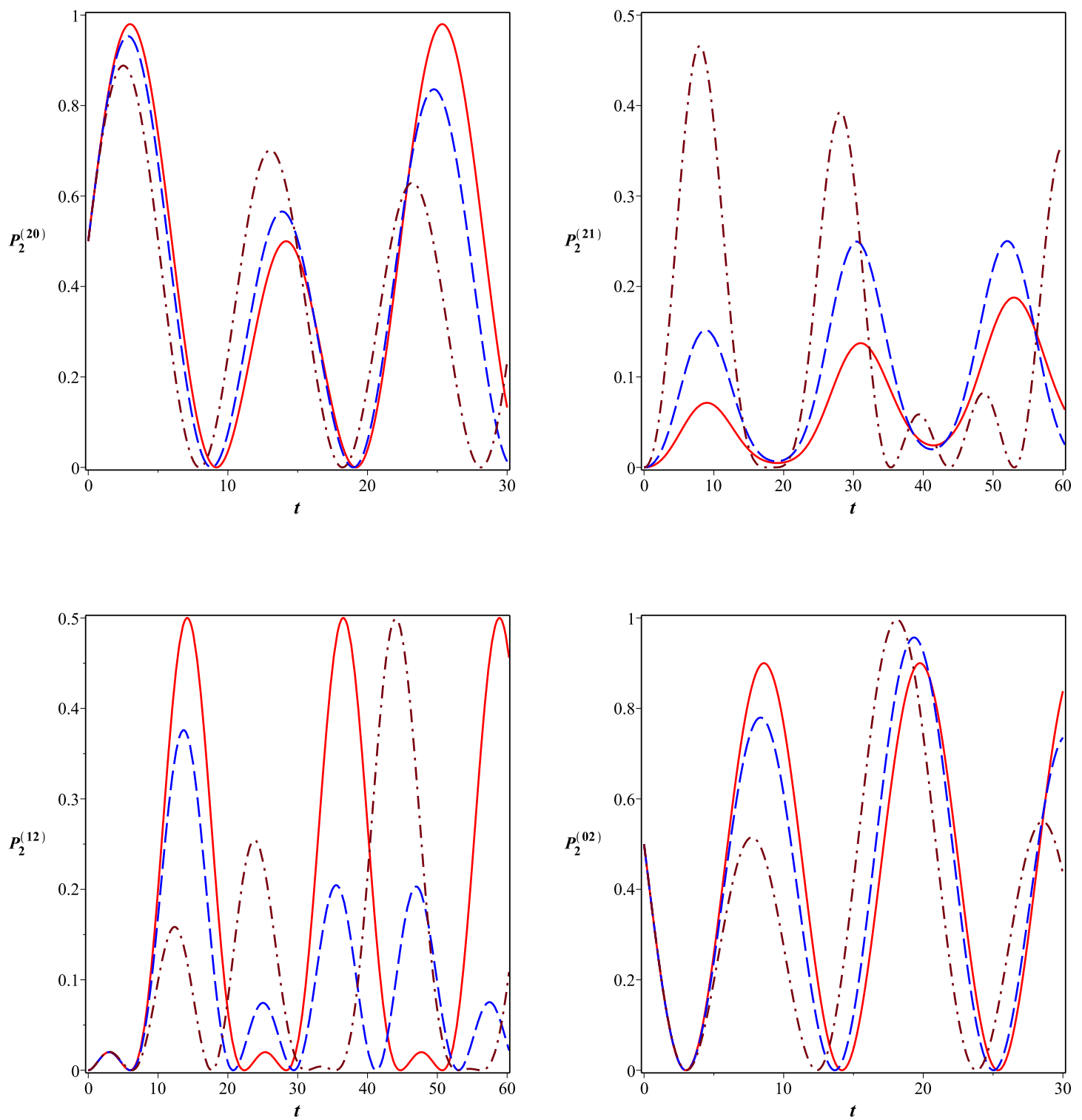

Fig. 4. The probabilities that the system exists in the Bell-like state $\left|B_{2}\right\rangle$ corresponding to the initial states $|2\rangle_{a}|0\rangle_{b}\left(P_{2}^{(20)}\right),|2\rangle_{a}|1\rangle_{b}\left(P_{2}^{(21)}\right),|1\rangle_{a}|2\rangle_{b}\left(P_{2}^{(12)}\right)$ and $|0\rangle_{a}|2\rangle_{b}\left(P_{2}^{(02)}\right)$ with $\alpha=\pi / 25, \varepsilon=\pi / 25$. Solid curve is for $\beta=0$, dashed curve is for $\beta=\pi / 50$ and dashed dotted curve is for $\beta=\pi / 25$. The time is scaled in $1 / \chi$ units.

The figures from Fig. 3 to Fig. 10 show the probabilities $P_{i}^{(20)}, P_{i}^{(21)}, P_{i}^{(12)}$ and $P_{i}^{(02)}$ of the cases when the system exists in the Bell-like states $\left|B_{i}\right\rangle(\mathrm{i}=1,2,3, \ldots, 8)$ corresponding to the initial states $|2\rangle_{a}|0\rangle_{b},|2\rangle_{a}|1\rangle_{b},|1\rangle_{a}|2\rangle_{b}$ and $|0\rangle_{a}|2\rangle_{b}$, respectively. For the initial state $|2\rangle_{a}|0\rangle_{b}$, when $\beta=0$, we again obtain the probabilities that the system existing in the states $\left|B_{i}\right\rangle$ pumped by an 
external coherent field [14]. When $\beta=\alpha$, we also achieve the probabilities of the case when the system exists in the states $\left|B_{i}\right\rangle$ pumped by two external coherent fields that have the same strength [28].
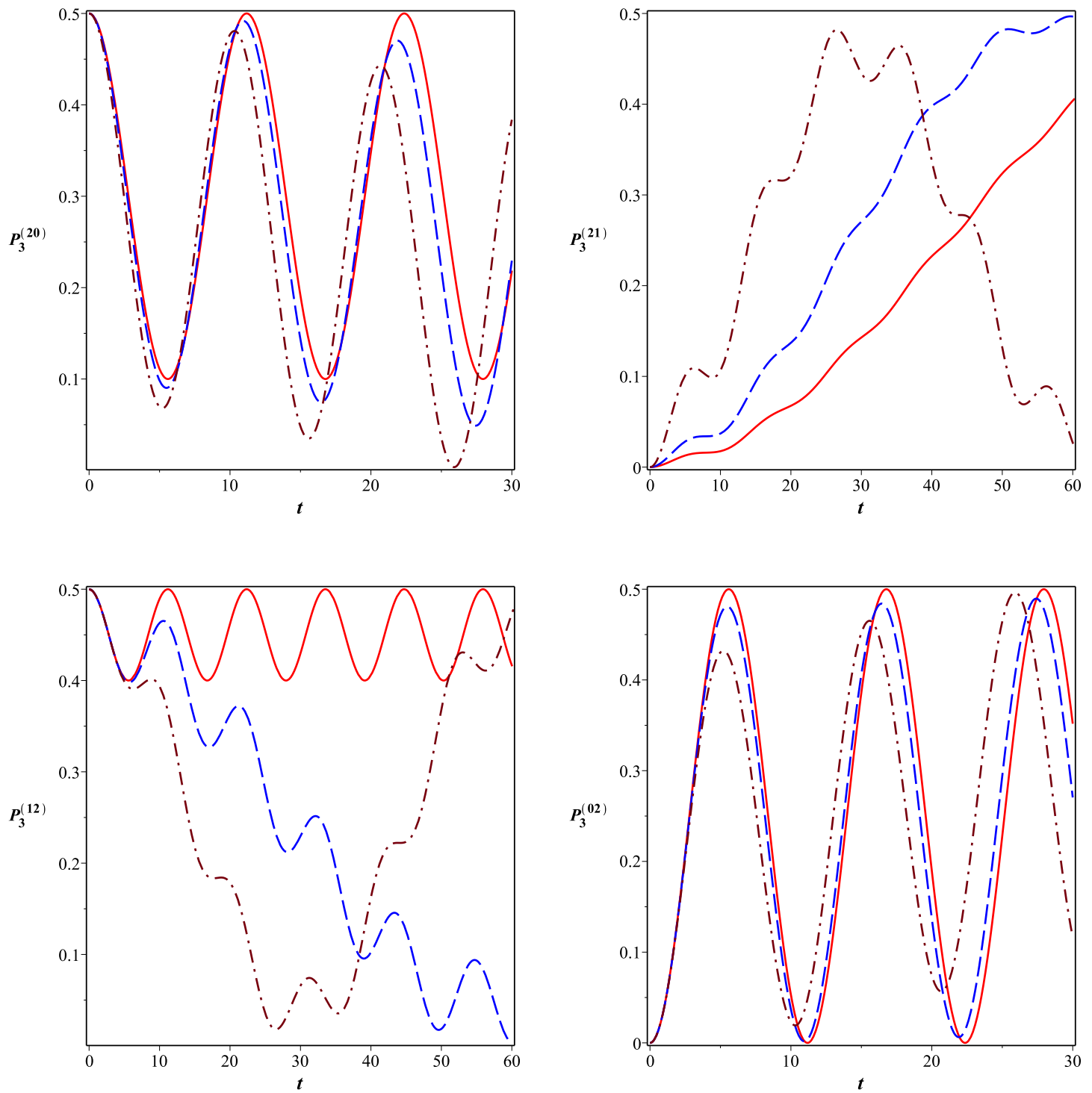

Fig. 5. The probabilities that the system exists in the Bell-like $\left|B_{3}\right\rangle$ corresponding to the initial states $|2\rangle_{a}|0\rangle_{b}\left(P_{3}^{(20)}\right),|2\rangle_{a}|1\rangle_{b}\left(P_{3}^{(21)}\right),|1\rangle_{a}|2\rangle_{b}\left(P_{3}^{(12)}\right)$ and $|0\rangle_{a}|2\rangle_{b}\left(P_{3}^{(02)}\right)$ with $\alpha=\pi / 25, \varepsilon=\pi / 25$. Solid curve is for $\beta=0$, dashed curve is for $\beta=\pi / 50$ and dashed dotted curve is for $\beta=\pi / 25$. The time is scaled in $1 / \chi$ units. 

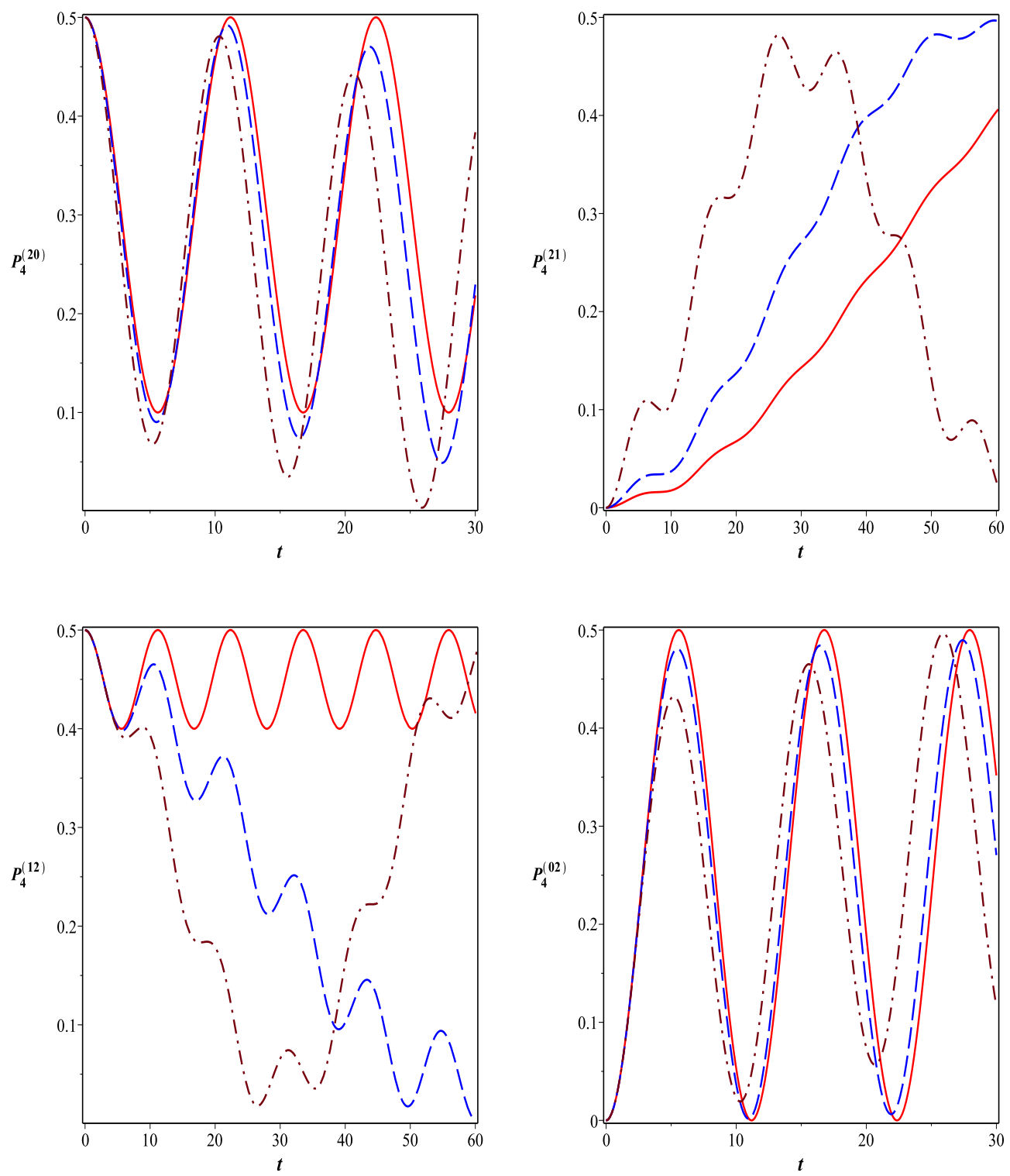

Fig. 6. The probabilities that the system exists in the Bell-like state $\left|B_{4}\right\rangle$ corresponding to the initial states $|2\rangle_{a}|0\rangle_{b}\left(P_{4}^{(20)}\right),|2\rangle_{a}|1\rangle_{b}\left(P_{4}^{(21)}\right),|1\rangle_{a}|2\rangle_{b}\left(P_{4}^{(12)}\right)$ and $|0\rangle_{a}|2\rangle_{b}\left(P_{4}^{(02)}\right)$ with $\alpha=\pi / 25, \varepsilon=\pi / 25$. Solid curve is for $\beta=0$, dashed curve is for $\beta=\pi / 50$ and dashed dotted curve is for $\beta=\pi / 25$. The time is scaled in $1 / \chi$ units. 

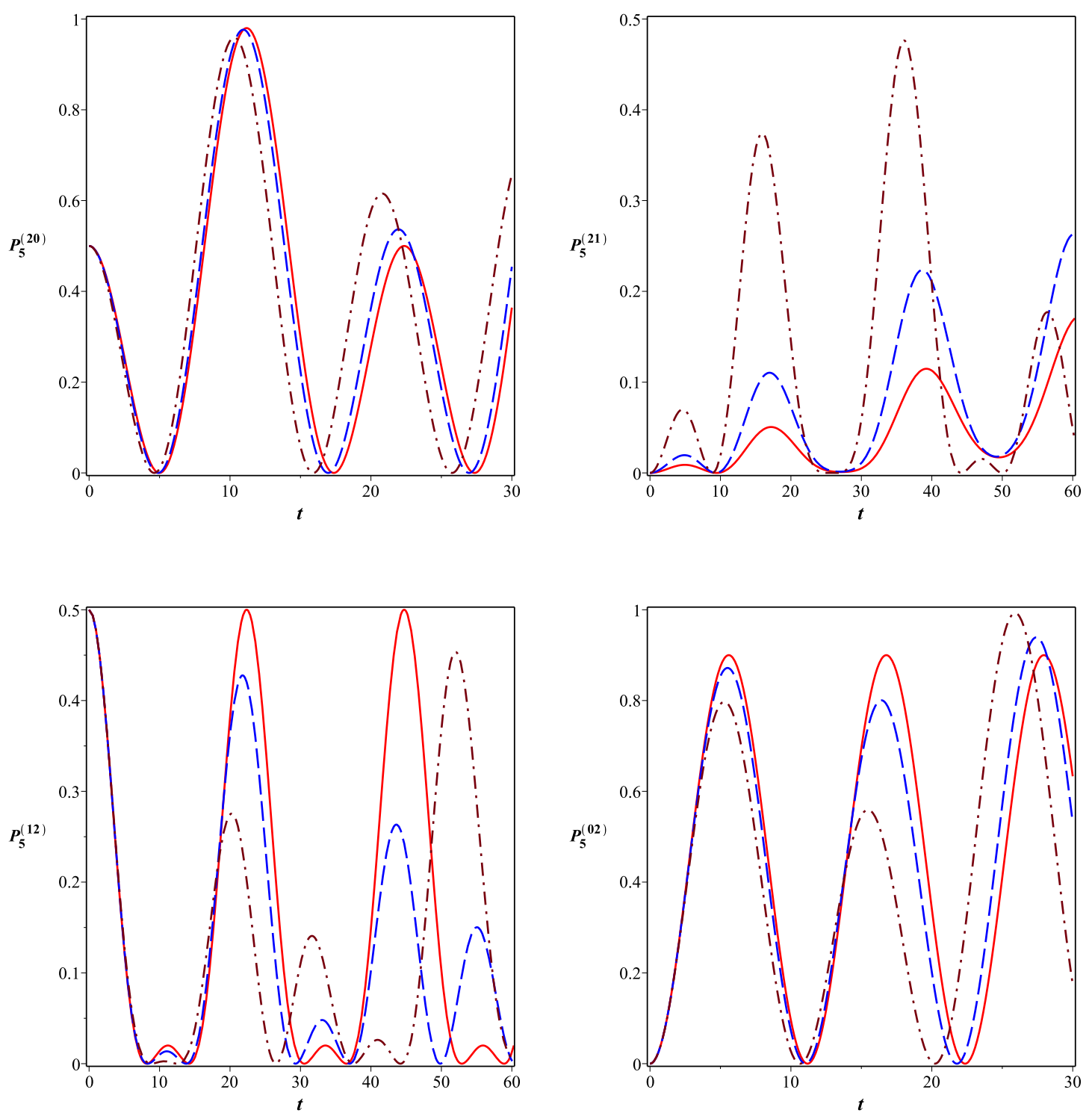

Fig. 7. The probabilities that the system exists in the Bell-like state $\left|B_{5}\right\rangle$ corresponding to the initial states $|2\rangle_{a}|0\rangle_{b}\left(P_{5}^{(20)}\right),|2\rangle_{a}|1\rangle_{b}\left(P_{5}^{(21)}\right),|1\rangle_{a}|2\rangle_{b}\left(P_{5}^{(12)}\right)$ and $|0\rangle_{a}|2\rangle_{b}\left(P_{5}^{(02)}\right)$ with $\alpha=\pi / 25, \varepsilon=\pi / 25$. Solid curve is for $\beta=0$, dashed curve is for $\beta=\pi / 50$ and dashed dotted curve is for $\beta=\pi / 25$. The time is scaled in $1 / \chi$ units. 

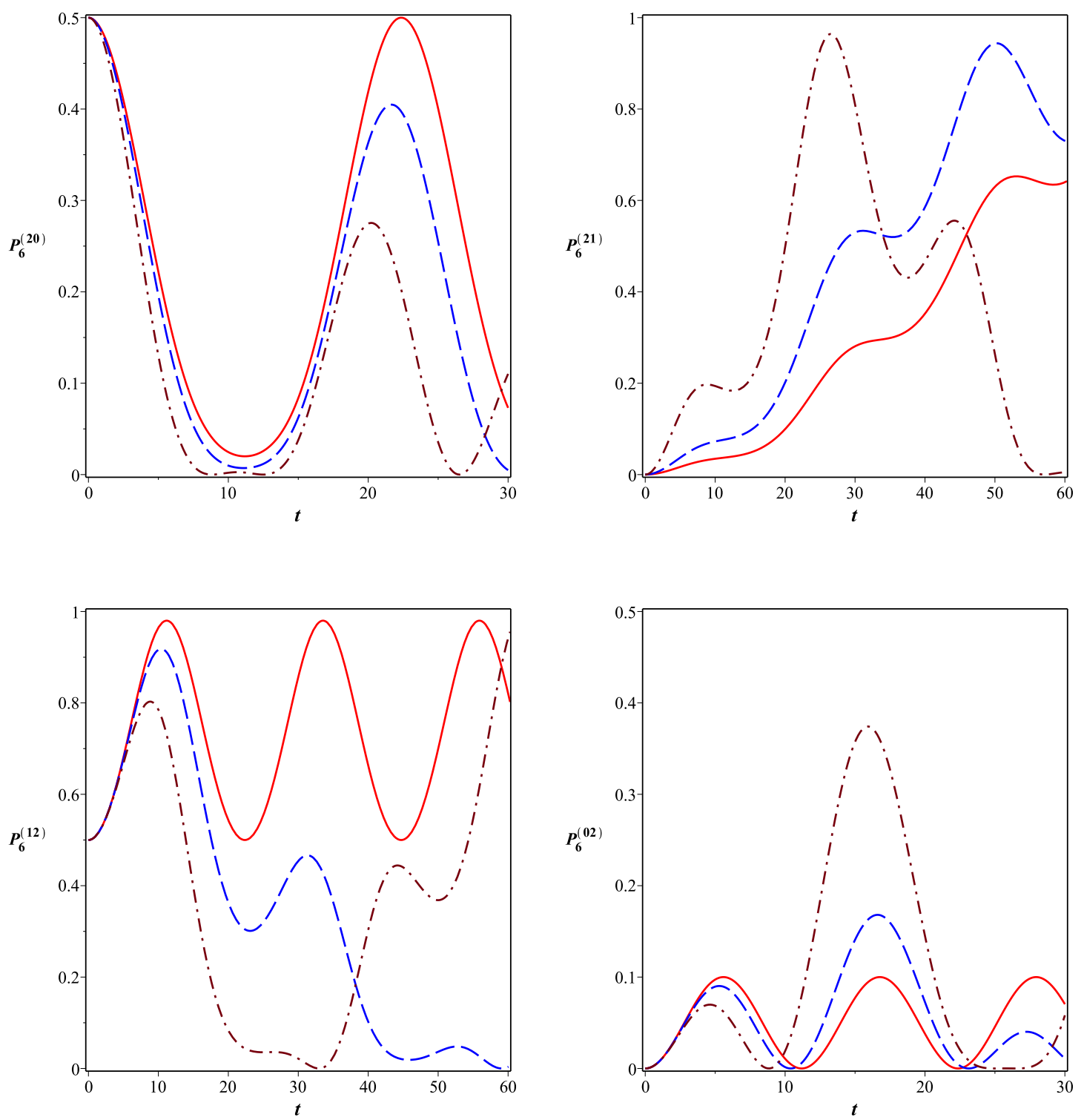

Fig. 8. The probabilities that the system exists in the Bell-like state $\left|B_{6}\right\rangle$ corresponding to the initial states $|2\rangle_{a}|0\rangle_{b}\left(P_{6}^{(20)}\right),|2\rangle_{a}|1\rangle_{b}\left(P_{6}^{(21)}\right),|1\rangle_{a}|2\rangle_{b}\left(P_{6}^{(12)}\right)$ and $|0\rangle_{a}|2\rangle_{b}\left(P_{6}^{(02)}\right)$ with $\alpha=\pi / 25, \varepsilon=\pi / 25$. Solid curve is for $\beta=0$, dashed curve is for $\beta=\pi / 50$ and dashed dotted curve is for $\beta=\pi / 25$. The time is scaled in $1 / \chi$ units.

Figures 3, 4, 7 and 9 show that the Bell-like states $\left|B_{1}\right\rangle,\left|B_{2}\right\rangle,\left|B_{5}\right\rangle$ and $\left|B_{7}\right\rangle$ can be generated when the initial states are $|2\rangle_{a}|0\rangle_{b}$ and $|0\rangle_{a}|2\rangle_{b}$, but they cannot be created when the initial states 

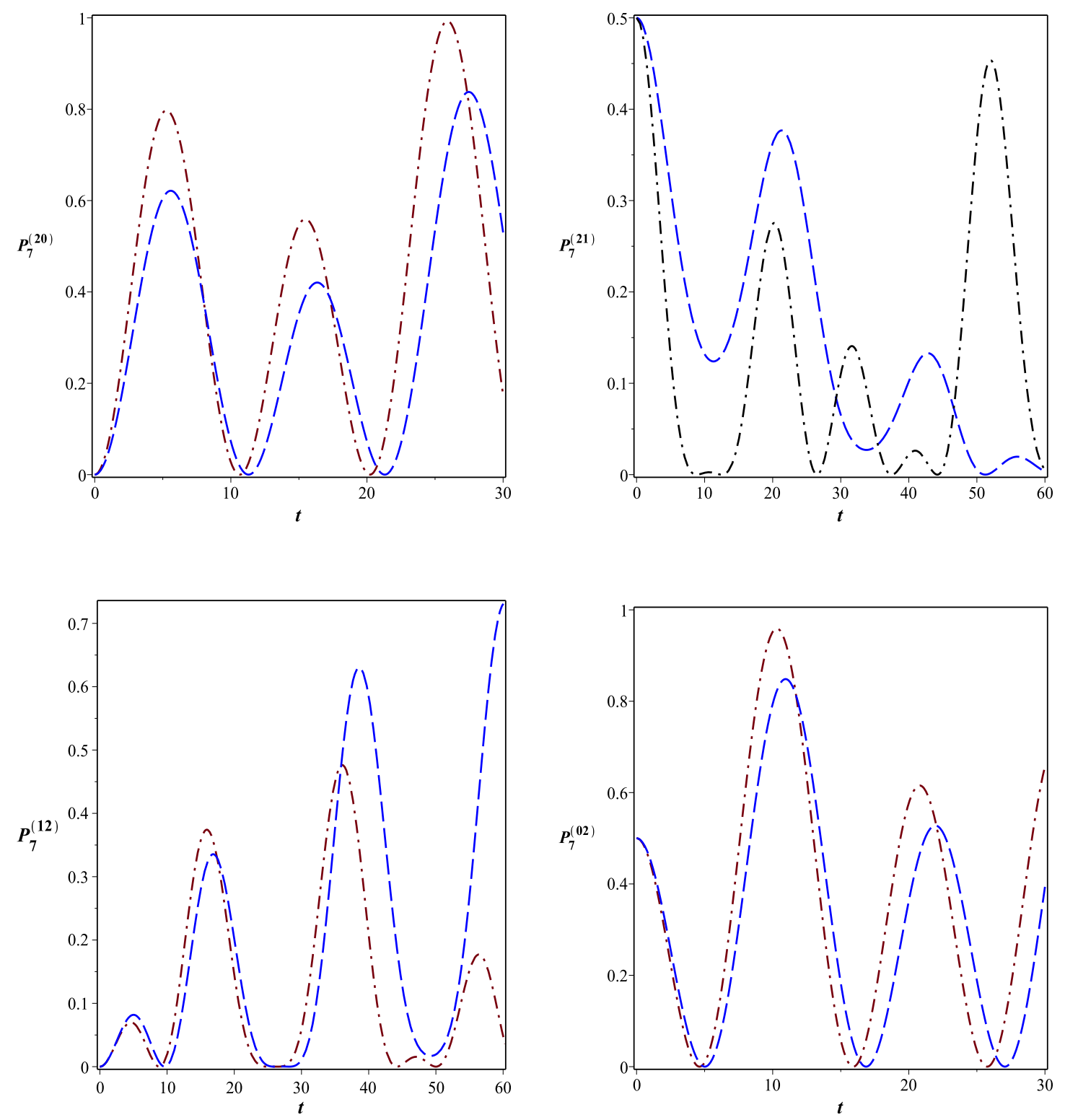

Fig. 9. The probabilities to the system existing in the Bell-like state $\left|B_{7}\right\rangle$ corresponding to the initial states $|2\rangle_{a}|0\rangle_{b}\left(P_{7}^{(20)}\right),|2\rangle_{a}|1\rangle_{b}\left(P_{7}^{(21)}\right),|1\rangle_{a}|2\rangle_{b}\left(P_{7}^{(12)}\right)$ and $|0\rangle_{a}|2\rangle_{b}\left(P_{7}^{(02)}\right)$ with $\alpha=\pi / 25, \varepsilon=\pi / 25$. Dashed curve is for $\beta=\pi / 50$ and dashed dotted curve is for $\beta=\pi / 25$. The time is scaled in $1 / \chi$ units.

are $|2\rangle_{a}|1\rangle_{b},|1\rangle_{a}|2\rangle_{b}$. When $\beta$ grows more and more, the largest peaks of probabilities $P_{1}^{(20)}$, $P_{2}^{(02)}, P_{5}^{(02)}, P_{7}^{(20)}$ and $P_{7}^{(02)}$ are also increasing approximately approaching one, whereas the largest peaks of probabilities $P_{1}^{(02)}, P_{2}^{(20)}$ and $P_{5}^{(20)}$ are decreasing and the positions of the maximum and 

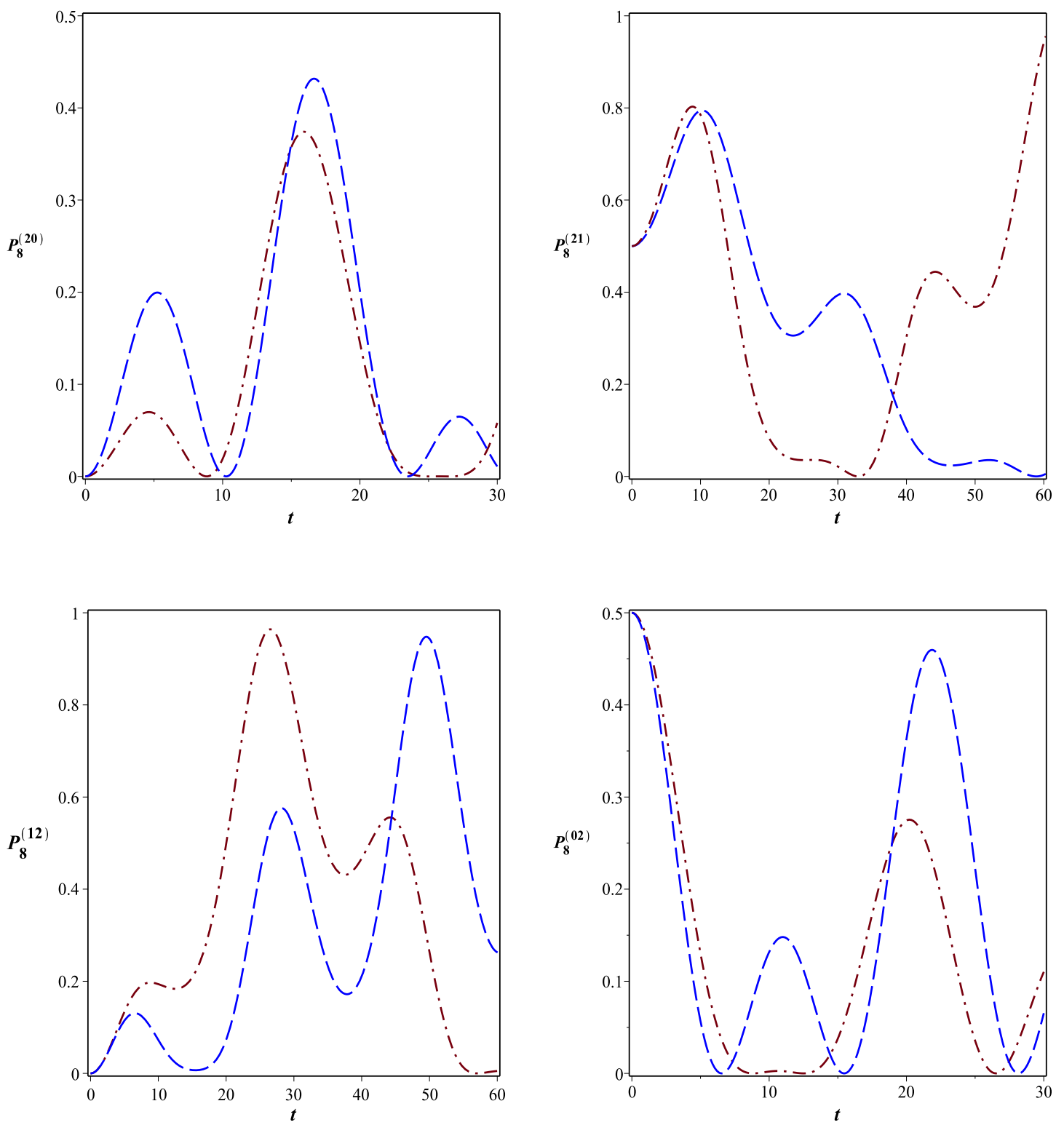

Fig. 10. The probabilities that the system exists in the Bell-like state $\left|B_{8}\right\rangle$ corresponding to the initial states $|2\rangle_{a}|0\rangle_{b}\left(P_{8}^{(20)}\right),|2\rangle_{a}|1\rangle_{b}\left(P_{8}^{(21)}\right),|1\rangle_{a}|2\rangle_{b}\left(P_{8}^{(12)}\right)$ and $|0\rangle_{a}|2\rangle_{b}\left(P_{8}^{(02)}\right)$ with $\alpha=\pi / 25, \varepsilon=\pi / 25$. Dashed curve is for $\beta=\pi / 50$ and dashed dotted curve is for $\beta=\pi / 25$. The time is scaled in $1 / \chi$ units.

minimum of the probabilities shift toward zero time. In contrast, the states $\left|B_{6}\right\rangle$ (Fig. 8) and $\left|B_{8}\right\rangle$ (Fig. 10) can create the Bell-like states corresponding to the initial states $|2\rangle_{a}|1\rangle_{b}$ and $|1\rangle_{a}|2\rangle_{b}$, but these states cannot create the Bell-like states corresponding to the initial states $|2\rangle_{a}|0\rangle_{b}$ and 
$|0\rangle_{a}|2\rangle_{b}$. We can see that the probabilities to the system existing in the states $\left|B_{3}\right\rangle$ (Fig. 5) and $\left|B_{4}\right\rangle$ (Fig. 6) are equal and they only reach the maximum value 0.5 .

\section{DISSIPATION CASE}

We can see that processes of damping can influence physical properties of our system and decrease the values of maximum of entanglement. Thus, we are going to focus on the effect of damping processes in the generation of Bell-like states. We suppose that $\kappa_{a}$ and $\kappa_{b}$ depict the losses of photons in two cavities $a$ and $b$, respectively. The photon loss corresponds to the annihilation of photons. Thus, we can deliberate the collapse operators with the forms as

$$
\begin{aligned}
& \hat{D_{a}}=\sqrt{2 \kappa_{a}} \hat{a}, \\
& \hat{D_{b}}=\sqrt{2 \kappa_{b}} \hat{b} .
\end{aligned}
$$

Here, the time-evolution of our system is characterized by a matrix of density $\hat{\rho}$. This matrix obeys the master equation in the following form

$$
\frac{d \hat{\rho}}{d t}=\hat{L} \hat{\rho}
$$

in which $\hat{L}$ is so-called Liouvillian superoperator. $\hat{L}$ in the Markov approximation can be written in the form

$$
\hat{L} \hat{\rho}=-i[\hat{H}, \hat{\rho}]+\hat{L}_{l} \hat{\rho},
$$

where $\hat{L}_{l}$ depicts the losses of photons in cavities. It is the loss term of the Liouvillian superoperator and has the following form

$$
\hat{L}_{l} \hat{\rho}=\hat{D}_{a} \hat{\rho} \hat{D}_{a}^{\dagger}-\frac{1}{2}\left(\hat{D}_{a}^{\dagger} \hat{D_{a}} \hat{\rho}-\hat{\rho} \hat{D}_{a}^{\dagger} \hat{D_{a}}\right)+\hat{D_{b}} \hat{\rho} \hat{D}_{b}^{\dagger}-\frac{1}{2}\left(\hat{D}_{b}^{\dagger} \hat{D_{b}} \hat{\rho}-\hat{\rho} \hat{D}_{b}^{\dagger} \hat{D_{b}}\right)
$$
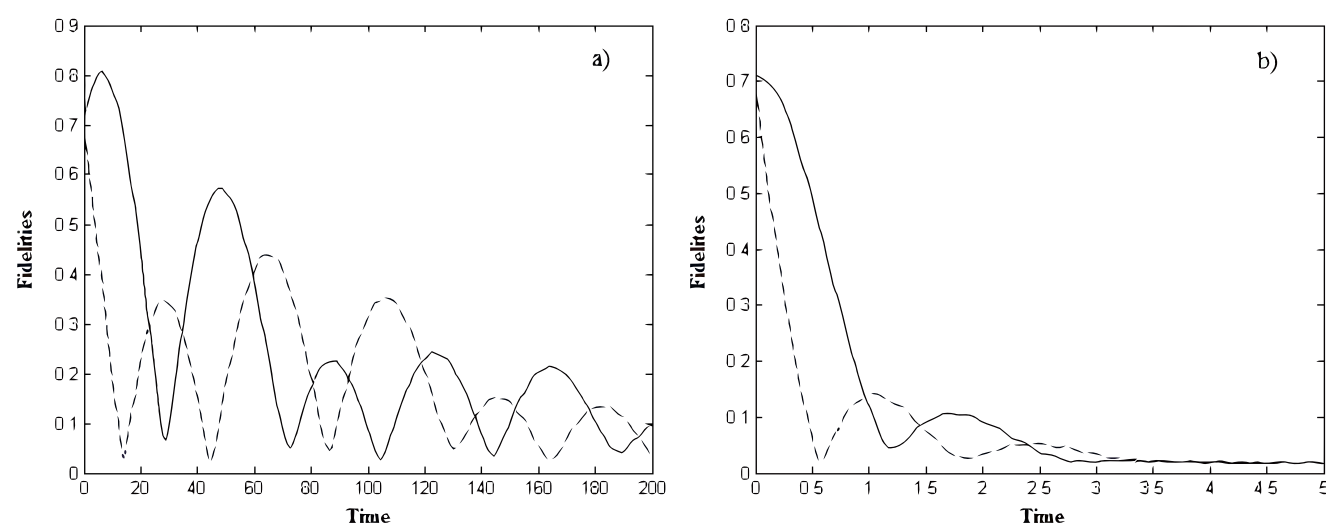

Fig. 11. Time-evolution of fidelities $\mathrm{F}$ corresponding to the Bell-like states $\left|B_{1}\right\rangle$ (solid lines) and $\left|B_{2}\right\rangle$ (dashed lines). The damping constants $\kappa_{a}=\kappa_{b}=\chi / 400$ (a) and $\kappa_{a}=$ $\kappa_{b}=\chi / 100$ (b). Other parameters are remained as in Fig. 1. Time unit is scalled in $1 / \chi$.

Figure 11 shows the fidelities corresponding to states $\left|B_{1}\right\rangle$ and $\left|B_{2}\right\rangle$ in the case $\varepsilon=\alpha=\beta$. We can see that the fidelities decrease when the damping constants $\kappa_{a}$ and $\kappa_{b}$ increase. However, 
in both cases $\kappa_{a}=\kappa_{b}=\chi / 400$ and $\kappa_{a}=\kappa_{b}=\chi / 100$, the variabilities of fidelities of state $\left|B_{1}\right\rangle$ and state $\left|B_{2}\right\rangle$ are opposite approximately. On the other hand, if we decrease the interaction constant between two modes $\epsilon$ to the value of $\epsilon=\frac{\alpha}{4}=\frac{\beta}{4}$, the values of fidelities increase appreciably that is shown in Fig. 12. The fidelities even have the high values that are equal to unit approximally in several moments of time.
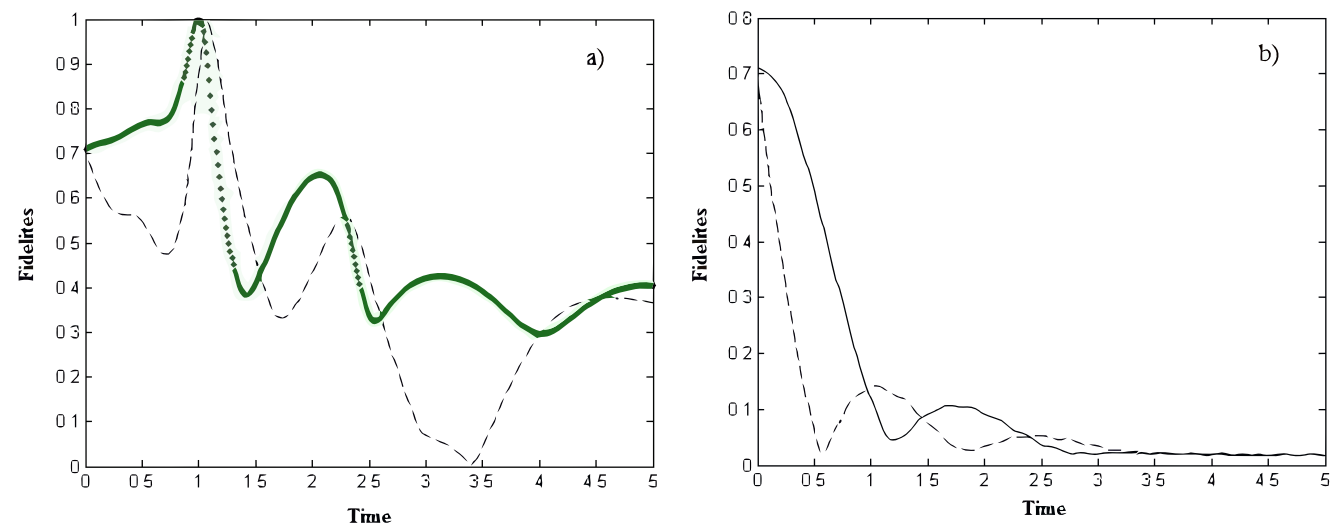

Fig. 12. Time-evolution of fidelities $\mathrm{F}$ corresponding to the Bell-like states $\left|B_{1}\right\rangle$ (solid lines) and $\left|B_{2}\right\rangle$ (dashed lines). The damping constants $\kappa_{a}=\kappa_{b}=\chi / 100$ in the case $\varepsilon=$ $\frac{\alpha}{4}=\frac{\beta}{4}$ (a) and $\varepsilon=\alpha=\beta$ (b). Time unit is scalled in $1 / \chi$.

\section{CONCLUSIONS}

In this paper, we have studied model of the Kerr-like nonlinear coupler including two nonlinear oscillators that are nonlinearly coupled to each other and were pumped in two modes by two external coherent fields. By using the formalism of NQS, we have obtained the time-evolution of system with all four initial conditions $|2\rangle_{a}|0\rangle_{b},|2\rangle_{a}|1\rangle_{b},|1\rangle_{a}|2\rangle_{b}$ and $|0\rangle_{a}|2\rangle_{b}$. It has been shown that most states $\left|B_{i}\right\rangle$ except for $\left|B_{3}\right\rangle$ and $\left|B_{4}\right\rangle$, which can be generated at least one maximally entangled state corresponding to certain initial states. Moreover, when parameter $\beta$ changes, the maximum values and positions of the probabilities for the system to exist in the Bell-like states also change. In addition, when the damping constant increases, , the fidelity decreases. Especially, the value of fidelity even is approximately equal to unit in some moments of time.

\section{ACKNOWLEDGMENT}

This research is funded by Vietnam National Foundation for Science and Technology Development (NAFOSTED) under grant number 103.03-2017.28.

\section{REFERENCES}

[1] A. Steane, Rep. Prog. Phys. 61 (1998) 117.

[2] R. Horodecki, P. Horodecki, M. Horodecki and K. Horodecki, Rev. Mod. Phys. 81 (2009) 865.

[3] J. I. Chirac and P. Zoller, Phys. Rev. Lett. 74 (1995) 4091.

[4] D. Loss and D. P. DiVincenzo, Phys. Rev. A 57 (1998) 120.

[5] J. Vidal, G. Palacios and C. Aslangul, Phys. Rev. A 70 (2004) 062304. 
[6] D. Bouwmeester, A. Ekert and A. Zeilinger, The Physics of Quantum Information, Berlin Springer, Press, 2000.

[7] D. Achilles, C. Silberhorn, C. Sliwa, K. Banaszek, I. A. Walmsley, M. J. Fitch, B. C. Jacobs, T. B. Pittman and J. D. Franson, J. Mod. Opt. 51 (2004) 1499.

[8] A. J. Miller, S. W. Nam, J. M. Martinis and A. V. Sergienko, App. Phys. Lett. 83 (2003) 791.

[9] M. Hamar, J. Perina Jr, O. Haderka and V. Michalek, Phys. Rev. A 81 (2010) 043827.

[10] J. Kim, S. Takeuchi, Y. Yamamoto and H. H. Hogue, App. Phys. Lett. 74 (1999) 902.

[11] M. Bondani, A. Allevi and A. Andreoni, Adv. Sci. Lett. 2 (2009) 463.

[12] A. Kowalewska-Kudlaszyk and W. Leonski, Progress in Optics 56 (2011) 131.

[13] A. Miranowicz and W. Leonski, J. Phys. B: At. Mol. Opt. Phys. 39 (2006) 1683.

[14] A. Kowalewska-Kudlaszyk and W. Leonski, Phy. Rev. A 73 (2006) 042318.

[15] A. Kowalewka - Kudlaszyk, W. Leonski and J. Perina Jr, Phys. Rev. A 83 (2011) 052326.

[16] J. K. Kalaga, A. Kowalewska-Kudlaszyk, W. Leonski and A. Barasinski, Phys. Rev. A 94 (2016) 032304.

[17] J. K. Kalaga and W. Leonski, Quantum Information Processing 16 (2017) 175.

[18] J. K. Kalaga, W. Leonski and J. Perina Jr., Phys. Rev. A 97 (2018) 042110.

[19] V. Perinova, A. Luks, J. Krepelka, W. Leonski and J. Perina Jr., Optics Communications 414 (2018) 146.

[20] J. S. Bell, Physics 1 (1964) 195.

[21] A. Einstein, B. Podolsky and N. Rosen, Phys. Rev. 47 (1935) 777.

[22] M. A. Nielsen and I. L. Chuang, Quantum Computation and Quantum Information, Cambridge University Press, 2000.

[23] M. Hayashi, Quantum information. An introduction, Berlin Heidelberg Springer Press, 2006.

[24] P. Lambropoulos and D. Petrosyan, Fundamentals of quantum optics and quantum information, Berlin Heidelberg Springer Press, 2007.

[25] L. Diosi, Lecture notes in physics, Berlin Heidelberg, Springer, 2007.

[26] G. Jaeger, Quantum information. An overview, Springer Science C Media, LLC New York, 2007.

[27] W. Leonski and V. Cao Long, Proc. SPIE 8697 (2012) 869728.

[28] V. Le Duc and V. Cao Long, Comput. Meth. Sci. Technol. 22 (2016) 245.

[29] G. Jaeger, Phys. Rev. A 18 (2009) 50. 